\title{
Desenvolvimento de algoritmos de migração baseados em propagação de ondas
}

* Lucas Ferreira Conz Eugenio (UFF), Leandro Di Bartolo (COGE/ON)

Copyright 2016, SBGf - Sociedade Brasileira de Geofísica

Este texto foi preparado para a apresentação no VII Simpósio Brasileiro de Geofísica, Ouro Preto, 25 a 27 de outubro de 2016. Seu conteúdo foi revisado pelo Comitê de seus associados. É proibida a reprodução total ou parcial deste material para propósitos comerciais sem prévia autorização da SBGf.

\section{Resumo}

A migração é uma das últimas etapas da extensa cadeia de procedimentos realizados no processamento sísmico, esta técnica visa a correção de possíveis distorções que ocorrem na imagem dos refletores em subsuperfície. Devido a atual dificuldade no imageamento de reservatórios de hidrocarbonetos em regiões com alta complexidade geológica, o método de migração reversa no tempo vem ganhando destaque através dos resultados satisfatórios nesses tipos de regiões. O presente trabalho tem como objetivo o desenvolvimento de algoritmos de migração reversa no tempo e sua aplicação em dados sísmicos sintéticos. A partir da análise dos resultados, verifica-se que o algoritmo desenvolvido foi capaz de reproduzir fielmente as condições geológicas dos modelos em subsuperfície.

\section{Introdução}

Os levantamentos sísmicos de reflexão correspondem a técnica da geofísica aplicada a prospecção de hidrocarbonetos mais largamente utilizada e bem conhecida. O sucesso deste método deve-se ao fato de que os dados brutos são processados de modo a produzir uma seção sísmica que representa uma imagem da estrutura geológica. A teoria deste método consiste na propagação de ondas sísmicas em subsuperfície e no registro das ondas que são refletidas por uma interface geológica. Interface geológica é definida pelo contato entre tipos de litologias diferentes, com propriedades físicas distintas. Ao encontrar essas interfaces, parte da onda sísmica irá ser refletida e parte será propagada, respeitando a Lei de Snell. Em uma aquisição, cada receptor irá registrar estas reflexões em diferentes tempos, de acordo com sua geometria. O painel gerado por esses registros é denominado de sismograma. No entanto, os sismogramas não são compostos apenas de reflexões geológicas. Há uma grande quantidade de ruídos aleatórios e coerentes que contaminam o sinal sísmico e que devem ser retirados.

O processamento sísmico corresponde ao conjunto de etapas que visa retirar os ruídos dos dados adquiridos e aumentar a relação sinal/ruído dos sismogramas. Os tipos de processamentos variam conforme o ambiente de aquisição, ou seja, existem técnicas de processamento utilizados especificamente para ambientes terrestres e para marinhos. A migração é uma das últimas etapas dentro destes processamentos e pode ser entendida como o conjunto de procedimentos pelos quais os campos de onda registrados são transformados, através de metodologias adequadas, em imagens corretamente posicionadas dos refletores em subsuperfície. Ela pode ser realizada em dados que já foram empilhados ou em dados pré-empilhamento. No presente trabalho, a migração será realizada em dados sintéticos préempilhamento.

Um dos métodos de maior destaque atualmente, seja na área acadêmica ou na indústria, é a migração reversa no tempo. A sua importância se explica em virtude de ser este um dos métodos mais poderosos disponíveis, onde não há restrições quanto a geologia em subsuperfície, como ocorre com outros métodos de migração. No entanto, sua utilização deve ser restrita a regiões de alta complexidade geológica visto que seu custo computacional é alto, especialmente em dados préempilhamento. Este método pode ser dividido em duas etapas: a primeira etapa consiste na propagação das ondas sísmicas no sentido direto (descendente); a segunda etapa corresponde à propagação do campo de ondas registrado nos sismogramas no sentido inverso (depropagação). $\mathrm{Na}$ segunda etapa é utilizada uma condição específica de imagem (Chang et al., 1986), que irá gerar a seção sísmica final migrada. Além disso, será necessário obter um sismograma sintético através de uma modelagem da equação acústica da onda.

Portanto, será necessário o desenvolvimento de dois algoritmos, o primeiro que será responsável pela propagação direta das ondas sísmicas e o segundo que irá depropagar a energia registrada nos sismogramas sintéticos e aplicar a condição de imagem utilizada. Cabe ressaltar que os dois algoritmos necessitam de um modelo de velocidades das camadas em subsuperfície. $\mathrm{Na}$ primeira seção do trabalho será apresentada a equação da onda utilizada para a modelagem, em seguida será apresentado o método utilizado para resolver a equação computacionalmente. Feito isso, será explicado o conceito de migração reversa no tempo e as condições de imagem utilizadas. Finalmente, serão apresentados alguns resultados da aplicação dos algoritmos de migração gerados e as conclusões obtidas a partir da análise destes resultados.

\section{Equação da Onda}

A equação de onda utilizada neste trabalho, que descreve bem a propagação de ondas acústicas compressionais (ondas $\mathrm{P}$ ) em meios isotrópicos e com densidade constante é,

$$
\nabla^{2} p-\frac{1}{c^{2}} \frac{\partial^{2} p}{\partial t^{2}}=-\rho s
$$


onde t é o tempo, $\rho$ é a densidade do meio, $s$ é a fonte sísmica, c é a velocidade de propagação do meio e p o campo de pressões acústicas.

No presente trabalho serão abordados apenas esquemas em duas dimensões, então a equação 1 se transforma em

$$
\frac{\partial^{2} p}{\partial t^{2}}=c^{2}\left(\frac{\partial^{2} p}{\partial x^{2}}+\frac{\partial^{2} p}{\partial z^{2}}\right)+c^{2} \rho s,
$$

onde $x$ e $z$ correspondem as componentes espaciais em metros.

\section{Método de Diferenças Finitas (MDF)}

Para resolver esta equação computacionalmente, foi necessário utilizar o método de diferenças finitas para aproximar as derivadas parciais da equação 2. Foi utilizada uma malha simples (Fig. 1) para discretizar o domínio espacial em pontos igualmente espaçados nas duas direções ( $x$ e $z$ ). Em cada ponto da malha as propriedades do meio, como velocidade de propagação $c(x, z)$ e pressão acústica $p(x, z)$, são definidos.

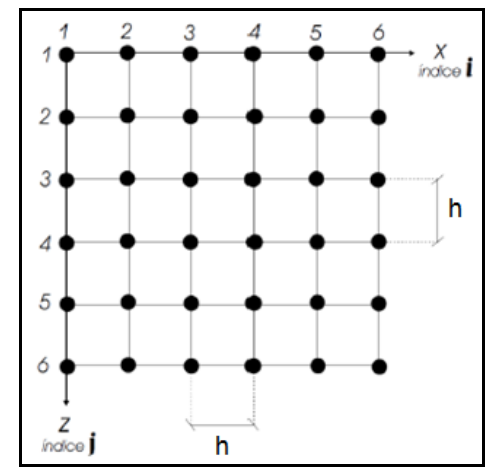

Figura 1 - Malha de Diferenças finitas utilizada (modificado de Di Bartolo, 2013).

As derivadas podem ser aproximadas por diferenças regressivas, progressivas ou centrais. Para melhor entendimento, o significado geométrico das diferenças finitas em uma dimensão pode ser visualizado na figura 2.

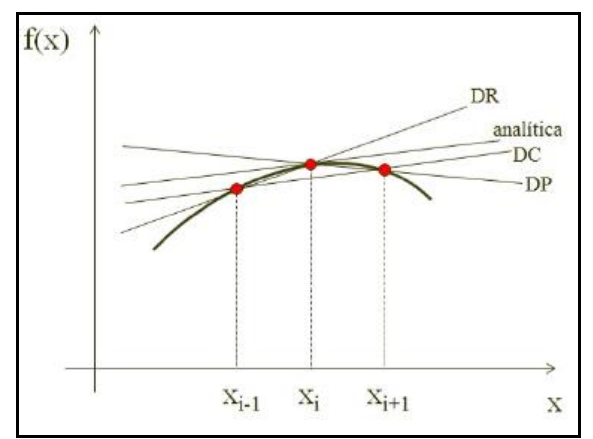

Figura 2 - Significado geométrico das aproximações em 1D. DR: Diferença Regressiva; DC: Diferença Central; DP: Diferença Progressiva (Di Bartolo, 2013).
No caso da diferença regressiva, a derivada é aproximada utilizando o próprio ponto e o ponto imediatamente anterior. Na progressiva, é utilizado o próprio ponto e o ponto imediatamente posterior. Já na central, são utilizados os pontos imediatamente posterior e anterior ao ponto que se quer calcular. No presente trabalho, foi utilizado a aproximação por diferenças centrais, por este apresentar precisão maior em comparação com a resposta analítica (Fig. 2).

\section{Operadores de Diferenças Finitas}

Os operadores de diferenças finitas substituem os termos de derivadas parciais na equação 2 e geram as equações de diferenças do MDF. Estes operadores podem ser classificados quanto a sua ordem, quanto maior a ordem, maior é o número de pontos utilizado para aproximar as derivadas. No presente trabalho, os operadores utilizados foram

$$
\begin{gathered}
\frac{\partial^{2} p}{\partial t^{2}}=\frac{p^{k-1}-2 p^{k}+p^{k+1}}{\Delta t^{2}} \\
\frac{\partial^{2} p}{\partial x^{2}}=\frac{-p_{i-2}+16 p_{i-1}-30 p_{i}+16 p_{i+1}-p_{i+2}}{12 \Delta x^{2}}
\end{gathered}
$$

onde $\Delta \mathrm{t}$ é o deslocamento da marcha no tempo e $\Delta \mathrm{x}=\Delta \mathrm{z}=\mathrm{h}$ é o espaçamento da malha simples em metros. A equação 3 representa operadores de $2^{\underline{a}}$ ordem no tempo e a equação 4 de $4^{a}$ ordem no espaço, que utilizam um stencil de 5 e 9 pontos, respectivamente. Aplicando estes operadores na equação 2 , obtém-se a equação 5 de diferenças finitas que será implementada.

$$
\begin{aligned}
& \frac{p_{i, j}^{k-1}-2 p_{i, j}^{k}+p_{i, j}^{k+1}}{\Delta t^{2}}=\frac{c_{i, j}^{2}}{12 h}\left(-p_{i-2, j}^{k}+16 p_{i-1, j}^{k}-30 p_{i, j}^{k}\right. \\
& +16 p_{i+1, j}^{k}-p_{i+2, j}^{k}-p_{i, j-2}^{k}+16 p_{i, j-1}^{k}-30 p_{i, j}^{k} \\
& \left.+16 p_{i, j+1}^{k}-p_{i, j+2}^{k}\right)+c_{i, j}^{2} \rho_{i, j} s_{i, j}^{k}
\end{aligned}
$$

\section{Metodologia}

A partir da discretização da equação 2, a seguinte metodologia foi utilizada: (1) gerar um modelo de velocidades (2) gerar um sismograma sintético a partir de uma modelagem direta; (3) realizar a migração reversa no tempo; (4) analisar os resultados obtidos. Nas etapas 2 e 3 os algoritmos foram desenvolvidos em Fortran 90 pelo autor.

\section{Modelagem Direta}

Em virtude do trabalho utilizar apenas dados sintéticos, foi necessário a geração de um sismograma sintético através de um algoritmo que simulasse uma aquisição sísmica. Para isso, a equação 2 foi discretizada utilizando os operadores MDF já vistos e a equação 5 foi implementada para gerar uma propagação de ondas. 
Para gerar o pulso sísmico, foi utilizada uma fonte sísmica Ricker com frequência de corte de $60 \mathrm{~Hz}$ (Fig. 3).

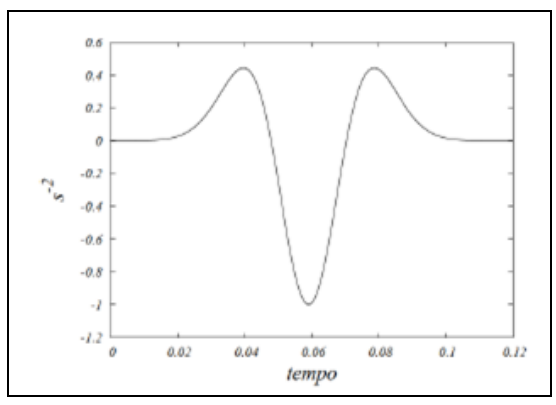

Figura 3 - Pulso sísmico injetado no algoritmo de propagação de ondas (Di Bartolo, 2013).

Além disso, uma geometria de aquisição de lanço lateral foi planejada. Para todos os modelos migrados, a geometria foi a mesma, com um cabo de 1000 metros de extensão, onde o offset mínimo foi de 25 metros e o espaçamento entre os receptores também foi de 25 metros. Cabe ressaltar que esta geometria foi incorporada ao algoritmo e o mesmo também foi implementado de forma a executar múltiplos tiros. O espaçamento entre tiros utilizado foi de 25 metros.

Depois de definir a equação da onda, a fonte sísmica e a geometria de aquisição utilizada, foram gerados dois modelos de velocidades (Fig. 4) com o objetivo de aplicar o algoritmo de modelagem direta desenvolvido. O primeiro modelo (Fig. 4a) é formado por quatro camadas paralelas com uma falha presente. O segundo modelo (Fig. 4b) é formado por 5 camadas, onde a primeira é uma camada com inclinação e existe uma pequena estruturação na quarta camada. Nos dois modelos as velocidades de propagação são maiores com o aumento da profundidade.

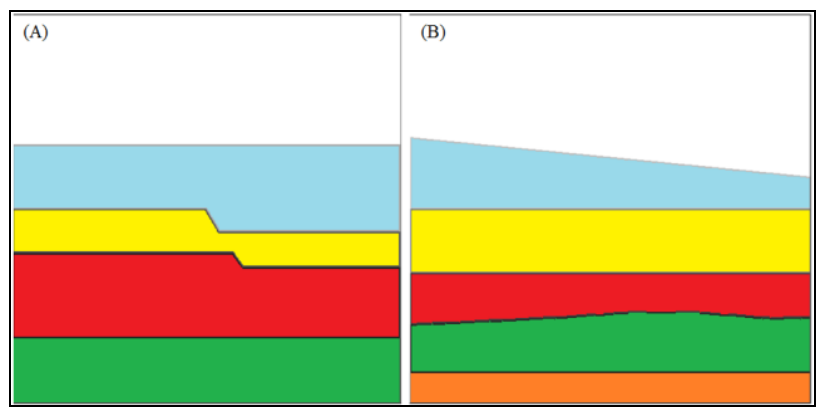

Figura 4 - Modelos de velocidade gerados. (A) modelo com falha; (B) modelo com estruturação na camada inferior.

Os modelos de velocidades foram fornecidos para o algoritmo de propagação de ondas e foi realizada a modelagem direta, com obtenção dos sismogramas sintéticos para cada modelo (Fig. 5). A aquisição foi realizada respeitando a geometria anteriormente mencionada, onde foram dados 120 tiros para cobrir toda a região do modelo, que tinha uma extensão de $3000 \times 3000$ metros. Foi utilizada uma malha simples com $601 \times 601$ pontos, com espaçamento de 5 metros.

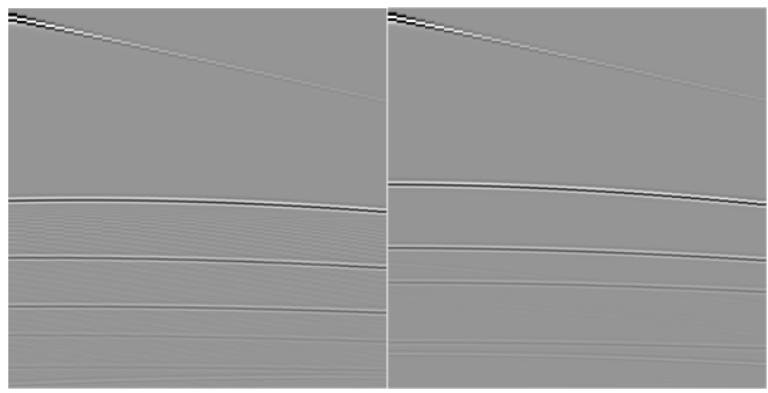

Figura 5 - Sismogramas sintéticos gerados. Sismograma para modelo de falha a esquerda e para modelo com estrutura nas camadas inferiores a direita.

\section{Migração Reversa no Tempo (MRT)}

Com os sismogramas sintéticos disponíveis e os modelos de velocidades, foi possível implementar os algoritmos de MRT. A migração reversa no tempo é constituída de duas etapas. A primeira etapa foi a obtenção de uma matriz de tempo de trânsito (MTT), que será utilizada na segunda etapa como condição de imagem. A MTT é uma matriz formada pelo tempo de trânsito do campo de ondas descendentes, isto é, apenas da onda direta. Sabe-se que a medida que a onda se propaga para maiores profundidades, ocorre a atenuação da amplitude da mesma. Portanto, o critério utilizado para registrar o tempo apenas da onda direta, foi o critério da amplitude máxima (Loewenthal \& Hu, 1991). A figura 6 ilustra a propagação de uma onda direta e a MTT correspondente.

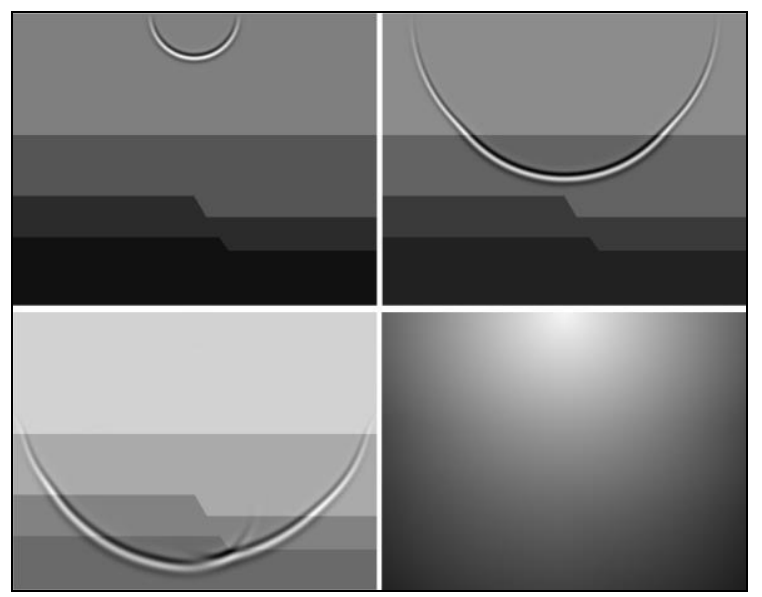

Figura 6 - Propagação de ondas no modelo da figura 4a. Notar a MTT com tempos contínuos no canto inferior direito.

Cada tiro dado durante a aquisição irá gerar uma MTT, bem como cada tiro terá um sismograma sintético associado. Com esses dois elementos, a segunda etapa da MRT pode ser realizada. Esta etapa consiste na depropagação do campo de ondas registrado nos receptores, ou seja, na propagação das energias presentes nos sismogramas no sentido inverso do tempo. Segundo Grey et. al, 1991, a depropagação é uma operação inversa da modelagem direta que visa desfazer os efeitos gerados pela mesma. 
O algoritmo de MRT irá avaliar, a cada passo de tempo, a condição de imagem por tempo de excitação (Fig. 7). A depropagação será feita do tempo final ao tempo inicial da modelagem direta, quando o tempo da depropagação for igual a algum tempo da MTT, a condição de imagem é satisfeita e o ponto é considerado um ponto imagem.

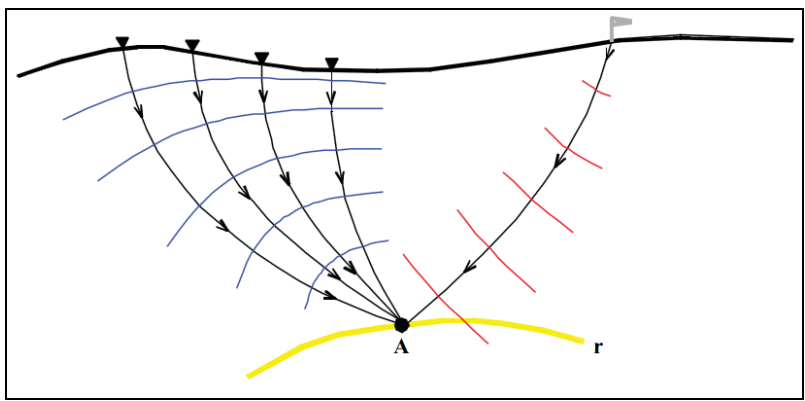

Figura 7 - Esquema da condição de imagem (Bulcão, 2004).

Feito isso, será gerada uma imagem para cada tiro dado durante a aquisição. A última etapa consiste em somar todas as imagens geradas a fim de obter uma última imagem do modelo. Essa soma nada mais é do que um processo de empilhamento, onde o interesse está em aumentar a resolução final da seção sísmica. Cabe ressaltar que cada tiro irá imagear uma determinada região, de acordo com a posição dos seus receptores. Ou seja, é esperado ao final do somatório que toda região do modelo seja adequadamente visualizada. A figura 8 mostra o processo de soma das imagens geradas para o modelo da figura $4 b$, onde pode-se verificar a melhoria da imagem a medida que uma maior quantidade de imagens é juntada à soma.

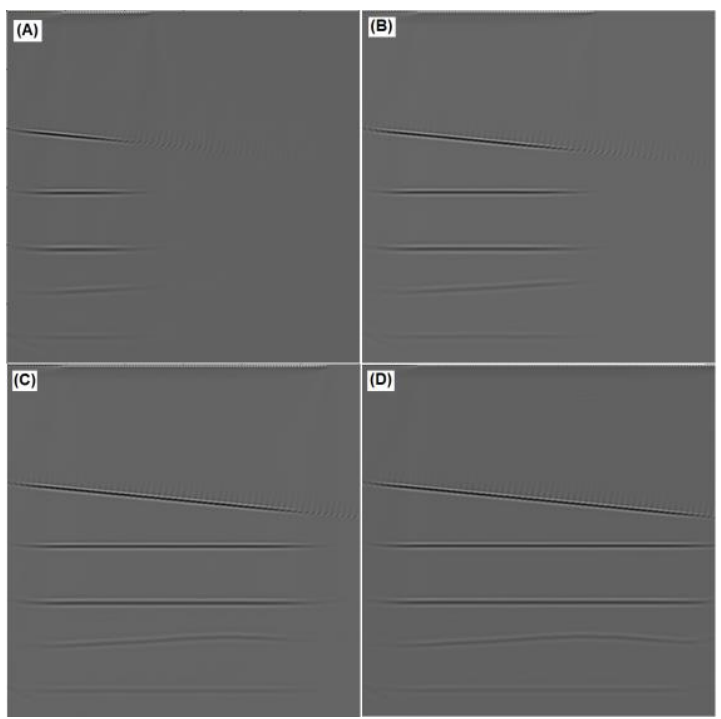

Figura 8 - Processo de soma de imagens. (A) soma de 30 imagens; (B) soma de 60 imagens; (C) soma de 90 imagens; (D) soma de 120 imagens.

O mesmo processo de soma foi realizado para o modelo da figura 4a. A imagem final obtida pode ser visualizada na figura 9 .

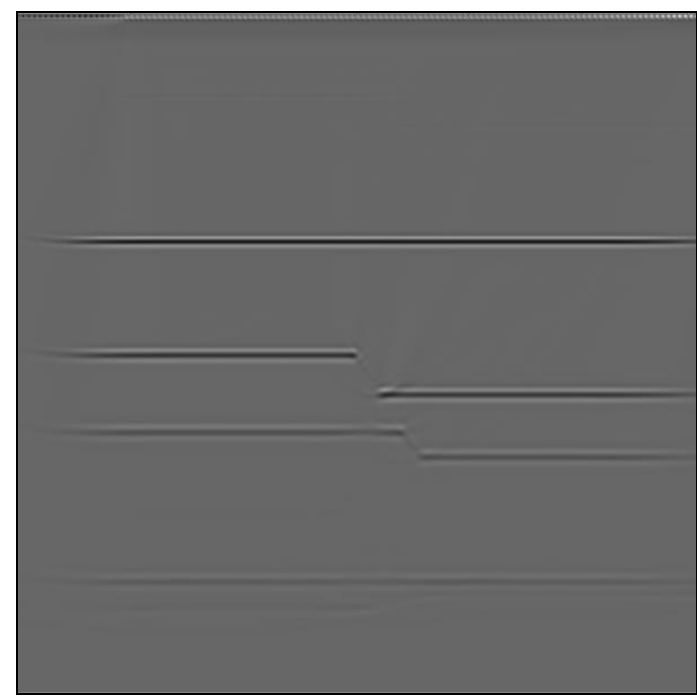

Figura 9 - Imagem obtida para o modelo 4a após 120 tiros.

\section{Conclusões}

Pode-se verificar que os algoritmos desenvolvidos durante o trabalho foram eficientes no processo de MRT, produzindo resultados satisfatórios dos modelos geológicos inicialmente propostos. Portanto, pode-se concluir que a MRT é uma ferramenta de grande qualidade para realização da migração sísmica. Ela é especialmente interessante pois não apresenta restrições quanto à geologia em subsuperfície, podendo ser utilizada em modelos altamente complexos. No entanto, uma desvantagem é seu alto custo computacional em tempo e memória, visto que é necessário gerar uma MTT e uma depropagação com aplicação da condição de imagem para cada tiro dado durante a aquisição sísmica.

\section{Agradecimentos}

Os autores agradecem à CNPq ao apoio dado a pesquisa de iniciação científica através da implementação de bolsa de estudos.

\section{Referências}

LOEWENTHAL, D., HU. L., 1991, "Two methods for computing the imaging condition for common-shot prestack migration", Geophysics, v. 56, pp. 378-381.

CHANG, W.F., McMECHAN, G., 1986, "Reverse-Time Migration of Offset Vertical Seismic Profiling Data Using the Excitation Time Imaging Condition", Geophysics, v. 51, pp. 67-84.

KEAREY, P.; BROOKS, M.; HILL, I., 2002, An Introduction to Geophysical Exploration, 3rd Edition. Blackwell Science: Oxford. 
DI BARTOLO, L., 2013, Introdução à Modelagem Sísmica utilizando o MDF. Universidade Estadual de Campinas. IV Semana de Inverno de Geofísica. Notas de Aula de Minicurso.

GRAY, S.H., ETGEN, J., DELLINGER, J., WHITMORE, D., 2001, "Seismic Migration Problems and Solutions", Geophysics, v. 66, pp. 1622-1640.

BULCÃO, A., 2004, Modelagem e Migração Reversa no Tempo Empregando Operadores Elásticos e Acústicos. Tese (Doutorado) - COPPE/UFRJ, Rio de Janeiro, Brasil. 